Ophthalmologe 2017 $\cdot 114: 496-497$

DOI 10.1007/s00347-017-0496-9

(c) Springer Medizin Verlag GmbH 2017

CrossMark

\section{P. Maier}

Klinik für Augenheilkunde, Universitätsklinikum Freiburg, Freiburg, Deutschland

\title{
Augenbeteiligung bei atopischer Dermatitis
}

Mit dem Leitthema „Augenbeteiligung bei atopischer Dermatitis" in diesem Heft wollen wir die Aufmerksamkeit unserer Leser auf ein häufiges dermatologisches Krankheitsbild, die Neurodermitis oder auch atopische Dermatitis, richten, das nicht selten auch charakteristische ophthalmologische Beschwerden und Veränderungen verursacht.

Um den Leser mit der zugrunde liegenden Hautkrankheit vertraut zu machen, beschreibt R. Fölster-Holst grundlegende Aspekte der atopischen Dermatitis aus Sicht der Dermatologin. Dabei geht sie zunächst auf die charakteristischen klinischen Hauterscheinungen und Beschwerden der Betroffenen ein, deren Lebensqualität durch den ausgeprägten Juckreiz und Stigmatisierungen häufig stark eingeschränkt wird. Daneben werden die verschiedenen Behandlungsmöglichkeiten an der Haut geschildert. Hinsichtlich dieser dermatologischen Therapiemaßnahmen sind die charakteristischen Basismaßnahmen zu einer Vermeidung der Allergenkonfrontation (Meidung der Triggerfaktoren, Patienten- und Elternschulungen etc.) und einer Verbesserung der Hautbarriere durch pflegende und rückfettende Externa entscheidend. Allerdings werden häufig im Falle einer Exazerbation der Hautmanifestationen topische und manchmal auch systemische antiinflammatorische Therapeutika notwendig.

T. Lapp et al. beschreiben die grundlegenden pathophysiologischen Prozesse, die dieser inflammatorischen (System-)Erkrankung mit den entsprechenden Ausprägungen am Auge ursächlich sind. In einem übersichtlichen Algorhithmus fassen die Autoren die pathophysiologischen Grundsätze mit den daraus resultierenden Symptomen und
Befunden zusammen, die schließlich entsprechende Behandlungsmaßnahmen notwendig machen.

》) In einem übersichtlichen
Algorithmus fassen Lapp et al.
pathophysiologische Grundsätze
samt Symptomen und Befunden
zusammen

Dabei wird erkennbar, dass es teils erworbene teils genetisch determinierte Störungen des immunologischen Gleichgewichts sind, die sowohl an der Haut als auch an der Augenoberfläche zu schwerwiegenden Veränderungen führen können. Eine mögliche gemeinsame Endstrecke könnte hierbei in einer Verminderung oder einem Fehlen von Filaggrin liegen. Beim Filaggrin handelt es sich um ein wichtiges Strukturprotein, das für die Intaktheit der Hautbarriere eine entscheidende Rolle spielt. Sowohl in der Haut als auch in der Kornea konnte bei Patienten mit atopischer Dermatitis eine verminderte bzw. fehlerhafte Filaggrinproduktion nachgewiesen werden.

Schließlich beschreiben P. Maier und T. Reinhard in einer Übersichtsarbeit die typischen klinischen Veränderungen an den Augenlidern, der Bindehaut und der Hornhaut. Dabei reicht das klinische Spektrum von einer milden Blepharitis mit Benetzungsstörung über eine chronisch vernarbende Keratokonjunktivitis mit Symblepharonbildung und Lidfehlstellungen bis hin zur kompletten Limbusstammzellinsuffizienz. Die Behandlung der ophthalmologischen Probleme reicht ähnlich wie die dermatologische Therapie von Basismaßnahmen wie der Lidrandpflege sowie antiallergischen 
und benetzenden Augentropfen über eine lokale immunmodulatorische $\mathrm{Be}$ handlung (z. B. topisches Pimecrolimus oder Ciclosporin A) bis zur systemischen Immunsuppression, wofür häufig Ciclosporin A zum Einsatz kommt, da dies für die Behandlung der atopischen Dermatitis zugelassen ist. Allerdings muss der behandelnde Augenarzt gerade bei der Lokaltherapie häufig auf einen hohen Evidenzgrad im Hinblick auf die Effektivität der Therapie verzichten und zu Off-label-Behandlungen greifen, da in der Literatur oft keine randomisierten kontrollierten Studien zu den verschiedenen Behandlungsmaßnahmen vorliegen. Neben den medikamentösen Therapien wird im Weiteren auch auf die nicht selten notwendigen chirurgischen Maßnahmen an der Augenoberfläche und den Augenlidern eingegangen.

Zusammenfassend wird aus den Arbeiten zum Leitthema ersichtlich, dass ein vielfältiges und multidisziplinäres Krankheitsbild vorliegt, das häufig ein gemeinsames Vorgehen von Allgemeinmedizinern, Dermatologen und Ophthalmologen notwendig macht, um für die Betroffenen die bestmögliche Versorgung zu erreichen.

Allen Lesern wünsche ich eine interessante und aufschlussreiche Lektüre.
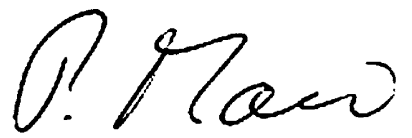

PD Dr. Philip Maier

\section{Korrespondenzadresse}

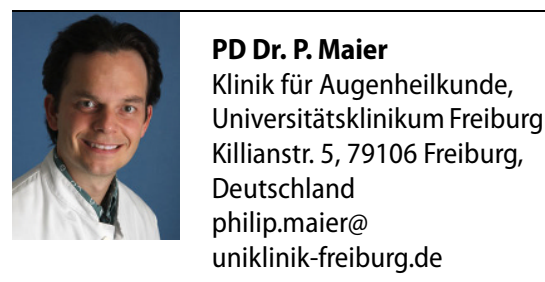

Interessenkonflikt. P. Maier gibt an, dass kein Interessenkonflikt besteht.

\section{Immer aktuell - Dank Online-First}

Warum warten, bis die Artikel im Heft abgedruckt sind, wenn Sie sie auch schon vorher lesen können?

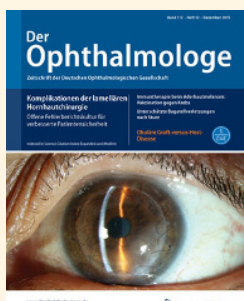

Lesen Sie unter www.springermedizin.de/der-ophthalmologe „Online First Artikel” die aktuellsten Artikel, die gerade online veröffentlicht wurden.

Folgende Übersichten, Originalien, Kasuistiken und „Bild und Fall“Beiträge wurden im vergangenen Monat online publiziert:

- Akute unilaterale Sehstörung einer 26-jährigen gesunden Frau.

S. Waibel, D. Sandner doi:10.1007/s00347-017-0491-1

- Akute, bilaterale Visusminderung bei einem Patienten mit IgA-Glomerulonephritis. J. Ehrhardt, F. Gelisken doi:10.1007/s00347-017-0492-0

- Makulopathie bei Grubenpapille. Morphologische Kriterien im SD-OCT. K. Wehrmann, S. Stumpfe, M. Pettenkofer et al. doi:10.1007/s00347-017-0490-21

- Unklare seröse Netzhautabhebung. M. Alnawaiseh, G. Wirths, L. Lahme, P. Nelis, N. Eter doi:10.1007/s00347-017-0493-z

- Management paraophthalmischer Aneurysmen. P. Bhogal, M. Aguilar Pérez, G. Sauder et al. doi:10.1007/s00347-017-0497-8

- Langzeitprognose der Peters-Anomalie. S. Reichl, D. Böhringer, O. Richter et al. doi:10.1007/s00347-017-0498-7

- Langzeitverlauf nach Keratoprothesen mit biologischer Haptik. K. Hille doi:10.1007/s00347-017-0503-1

- Seröse Amotio retinae bei Hyperviskositätssyndrom. V. C.Müller, N.Mihailovic, C. R. Clemens et al. doi:10.1007/s00347-017-0505-z

- Optische Kohärenztomographie bei seniler Retinoschisis. SD-OCT versus Ultraschalluntersuchung und Analyse des vitreoretinalen Übergangs. A. Bringewatt, S. Burzer, N. Feucht et al. doi:10.1007/s00347-017-0504-0

- Progrediente Ulzerationen der Periorbita. A. Frings, V. Frings, G. Geerling et al. doi:10.1007/s00347-017-0512-0

- Neu aufgetretene Visusminderung bei choroidalem Osteom. A. Schuh, P. Foerster, S. Priglinger et al. doi:10.1007/s00347-017-0514-y

- Auffälliger Papillenbefund bei einem 27-jährigen Patienten. K. Schöpfer, C. Scheib doi:10.1007/s00347-017-0507-x

- Ultraweitwinkelangiographie beim retinalen Venenverschluss. Wie groß ist groß genug? M. Storch, S. Bemme, M. Rehak et al. doi:10.1007/s00347-017-0513-z

Übrigens: Wussten Sie schon, dass die Artikel mit Angabe der DOI (digital object identifier) und dem Zusatz "epub ahead of print" bereits zitiert werden können? 